OPEN ACCESS

Edited by:

Subhash Verma,

Chaudhary Sarwan Kumar Himachal

Pradesh Krishi Vishvavidyalaya, India

Reviewed by:

Wolfgang Ludwig Köster,

University of Saskatchewan, Canada

Tongling Shan,

Chinese Academy of Agricultural

Sciences (CAAS), China

*Correspondence:

Fang Tang

tfalice@126.com

Jianjun Dai

daijianjun@njau.edu.cn

Specialty section:

This article was submitted to Veterinary Infectious Diseases,

a section of the journal

Frontiers in Veterinary Science

Received: 03 August 2020

Accepted: 30 October 2020

Published: 25 November 2020

Citation:

Li D, Qian X, Liu X, Sun Y, Ren J, Xue F, Liu Q, Tang F and Dai J (2020) orf6 and orf10 in Prophage phiv142-3

Enhance the Iron-Acquisition Ability and Resistance of Avian Pathogenic Escherichia coli Strain DE142 to

Serum. Front. Vet. Sci. 7:588708 doi: 10.3389/fvets.2020.588708

\section{orf6 and orf10 in Prophage phiv142-3 Enhance the Iron-Acquisition Ability and Resistance of Avian Pathogenic Escherichia coli Strain DE142 to Serum}

\author{
Dezhi Li ${ }^{1,2}$, Xinjie Qian ${ }^{2}$, Xinyuan Liu' ${ }^{2}$, Yu Sun ${ }^{2}$, Jianluan Ren ${ }^{2}$, Feng Xue ${ }^{2}$, Qing Liu ${ }^{1}$, \\ Fang Tang ${ }^{2 *}$ and Jianjun Dai ${ }^{2,3 *}$
}

\footnotetext{
${ }^{1}$ School of Medical Instrument and Food Engineering, University of Shanghai for Science and Technology, Shanghai, China, ${ }^{2}$ Ministry of Education Joint International Research Laboratory of Animal Health and Food Safety, Key Laboratory of Animal Bacteriology, Ministry of Agriculture, College of Veterinary Medicine, Nanjing Agricultural University, Nanjing, China, ${ }^{3}$ China Pharmaceutical University, Nanjing, China
}

Avian pathogenic Escherichia coli (APEC), an extraintestinal pathogenic E. coli (ExPEC), is the causative agent of avian colibacillosis, a disease that causes huge economic losses in the poultry industry and is characterized by infection through respiratory tract colonization followed by bacteraemia. A previous study in our lab demonstrated that phiv142-3 enhanced the survival ability of APEC strain DE142 in chickens serum. However, the mechanism of this affect has not been completely revealed. Here, we analyzed the transcriptional level of the prophage phiv142-3 region in DE142 when grown in chicken serum. Several upregulated genes attracted our attention, and a series of mutants were constructed. Deletion of orf6 or orf10 from phiv142-3 led to lower yields compared with WT after cultivation in serum for $10 \mathrm{~h}(P<0.05)$. Furthermore, avian infection assays showed that compared with WT, the bacterial loads in blood and heart tissue of chickens challenged with DE142 $\Delta$ orf6 were decreased to 3.9 and $13 \%$, while the bacterial burden in blood and heart from chickens infected with DE142 $\Delta$ orf10 was decreased to 7.2 and $8 \%$, respectively $(P<0.05)$. DE142 $\Delta$ orf6 showed an obviously attenuated growth rate in the logarithmic phase when cultured in iron-deficient medium, and the transcription level of the iutA gene decreased to $43 \%(P<0.05)$. The bactericidal assays showed that the survival of the mutant DE142 $\Delta$ orf10 was 60\% compared with WT in 50\% chicken serum. The K1 capsule-related genes (kpsF, kpsE, kpsC, and kpsM) were down-regulated nearly 2 -fold in DE142 $\Delta$ orf10 $(P<0.01)$. Together, these results suggested that orf6 affects growth by contributing to the uptake ability of iron, while orf10 increases resistance to serum by upregulating $\mathrm{K} 1$ capsule-related genes.

Keywords: avian pathogenic Escherichia coli, prophage, serum resistance, iron acquisition, colonization 


\section{INTRODUCTION}

The viruses that infect bacteria, known as bacteriophages (phages), are the most abundant biological entity on Earth, with an estimated global population of $10^{31}$ (1). They play a crucial role in controlling bacterial populations through phagemediated killing and the formation of prophages whose DNA inserts into the bacterial genome or is maintained as an episome after infecting bacteria. Most bacterial genomes harbor multiple prophages and contain up to $20 \%$ prophage sequences $(2,3)$. The role of these prophage genes in bacterial genomes remains largely unknown.

In lysogeny, the survival of prophages depends on the survival of the bacterial host in which it resides. Thus, from the perspective of evolution, it is advantageous for prophages to encode genes that promote fitness of the bacterial host. Through comparative genomics, pathogenic strains have been shown to harbor a larger proportion of prophage sequences than non-pathogenic strains (4). The greatest advantage associated with prophages is their ability to increase the resistance of the host to superinfection (5). Moreover, prophages can also alter many traits that increase host survival in different environments (6-8). For instance, it has been reported that after $\Phi$ Min27 integrates into the E. coli genome, the swimming motility of the lysogen is increased, allowing bacteria to move to high nutrient locations and avoid adverse environments (9). The lysogenic strain E. coli MG1655 ( $\Phi$ Min27) grows faster than MG1655 (without the prophage $\Phi$ Min27) (10). A lysogenic Streptococcus suis strain (with a prophage integrated into its genome) exhibited a faster growth rate and greater virulence (11). Similarly, Wang knocked out all nine prophages in $E$. coli K-12 BW25113 and found that the resulting strain showed decreased resistance to acid, osmotic and oxidative stresses (12). The Bor and Iss proteins are encoded by a prophage, which conferred resistance to lysogenic E. coli $(13,14)$. Recent studies have also pointed out that prophages increase the competitive fitness of lysogenic hosts and play important roles in bacterial diseases (15-18).

APEC is the causative agent of avian colibacillosis and causes huge economic losses in the poultry industry. The typical route of infection is respiratory tract colonization followed by bacteraemia (19). Resistance to serum appears to be an important factor for APEC infection $(20,21)$. Studies show that a number of virulence factors, such as outer membrane proteins (OMPs) and capsules, are associated with the complement resistance of $E$. coli $(20,22,23)$. In addition, assimilated iron is tightly bound to various proteins in animal blood, so serum is an iron-deficient condition for most bacteria $(24,25)$. However, iron, which is involved in many bacterial biological processes, is a crucial nutrient for most bacteria $(26,27)$. This low iron availability is another way that animals are protected against bacterial pathogens. APEC encode four types of siderophores involved in iron uptake systems to obtain iron, including enterobactin, salmochelins, yersiniabactin, and aerobactin. Corresponding receptors on the surface of cell membranes can bind to these sideophores and transfer iron-siderophore complexes into the cell (28).
TABLE 1 | Bacterial strains and plasmids used in this study.

\begin{tabular}{|c|c|c|}
\hline $\begin{array}{l}\text { Strains and } \\
\text { plasmids }\end{array}$ & Description & References \\
\hline DE142 & APEC strain, O2 serotype & This study \\
\hline DE142 $\Delta$ orf6 & orf6 deletion mutant & This study \\
\hline DE142 $\Delta$ orf6/orf6* & $\begin{array}{l}\text { Complementation strain, } \\
\text { containing pSTV28-orf6 plasmid }\end{array}$ & This study \\
\hline DE142 $\Delta$ orf6 $\Delta$ iutA & orf6 and iutA deletion mutant & This study \\
\hline DE142 $\Delta$ orf6/iutA* & $\begin{array}{l}\text { Complementation strain, } \\
\text { containing pSTV28-iutA plasmid }\end{array}$ & This study \\
\hline DE142 $\Delta$ orf10 & orf10 deletion mutant & This study \\
\hline DE142 $\Delta$ orf10/orf10* & $\begin{array}{l}\text { Complementation strain, } \\
\text { containing pSTV28-orf10 plasmid }\end{array}$ & This study \\
\hline DE142 $\Delta$ orf24 & orf24 deletion mutant & This study \\
\hline DE142 $\Delta$ orf40 & orf40 deletion mutant & This study \\
\hline DE142 $\Delta$ orf41 & orf41 deletion mutant & This study \\
\hline DE142A & $\begin{array}{l}\text { DE142, containing pUC19 } \\
\text { plasmid, Amp }\end{array}$ & This study \\
\hline DE142 $\Delta$ orf6C & $\begin{array}{l}\text { DE142 } 1 \text { orf6, containing pSTV28 } \\
\text { plasmid, Cm }\end{array}$ & This study \\
\hline pKD46 & Express $\lambda$ red recombinase, Amp & $(30)$ \\
\hline pKD4 & Template plasmid, Kan & (30) \\
\hline $\begin{array}{l}\text { pCP20 } \\
\text { pSTV28 }\end{array}$ & $\begin{array}{l}\text { Yeast Flp recombinase gene, FLP, } \\
\mathrm{Cm}, \mathrm{Amp} \\
\text { Expression using lac } \\
\text { promotor, Cm }\end{array}$ & $\begin{array}{l}\text { Takara } \\
\text { Takara }\end{array}$ \\
\hline pUC19 & Amp & Takara \\
\hline
\end{tabular}

A previous study in our lab found that prophage phiv142-3 enhanced the survival ability of APEC strain DE142 in chicken serum (29), but the mechanism of this effect is still unclear. The 54 genes in phiv142-3 from attL to attR were named orf1-orf54 according to the order of the locus. Therefore, the goal of this study was 2-fold. First, we aimed to determine which genes in phiv142-3 contribute to bacterial growth in serum and, if any genes were identified, to further characterize these genes. The work presented here described the use of qRT-PCR methods to screen the transcription level of genes in phiv142-3, and two knockout mutants were constructed to identify the role of the genes in vivo and in vitro.

\section{MATERIALS AND METHODS}

\section{Bacterial Strains, Plasmids, Primers, and Culture Conditions}

The bacterial strains and plasmids used in this study are summarized in Table 1, and oligonucleotide primers are listed in Table 2. Unless stated otherwise, all E. coli cultures were grown at $37^{\circ} \mathrm{C}$ in Luria-Bertani (LB) liquid medium with aeration. Appropriate antibiotics were added to the LB medium when necessary at the following concentrations: ampicillin (Amp, 100 $\mu \mathrm{g} \mathrm{mL}^{-1}$ ), kanamycin (Kan, $50 \mu \mathrm{g} \mathrm{mL}^{-1}$ ), and chloramphenicol $\left(\mathrm{Cm}, 30 \mu \mathrm{g} \mathrm{mL}^{-1}\right)$. 
TABLE 2 | Primers used in this study.

\begin{tabular}{|c|c|c|}
\hline Primer & Sequence $\left(5^{\prime}\right.$ to $\left.3^{\prime}\right)$ & Target gene \\
\hline $\mathrm{K} 1$ & CAGTCATAGCCGAATAGCCT & pKD4 \\
\hline K2 & CGGTGCCCTGAATGAACTGC & pKD4 \\
\hline Kt & CGGCCACAGTCGATGAATCC & pKD4 \\
\hline pKD46-F & GATACCGTCCGTTCTTCCTT & pKD46 \\
\hline pKD46-R & TGATGATACCGCCTGCCTTACT & \\
\hline pCP20-F & ATTGGGTACTGTGGGTTAGTGGTT & pCP20 \\
\hline pCP20-R & TTGGCTTATCCCAGGAATCTGTC & \\
\hline orf6Mu-F & $\begin{array}{l}\text { TICACAAATAAGCCGCCAACAA } \\
\text { GAAGACGGCATTAACATTAAAAT } \\
\text { AATTATATATGATGGTGTAGGCTG } \\
\text { GAGCTGCTTC }\end{array}$ & pKD4 \\
\hline orf6Mu-R & $\begin{array}{l}\text { AGGTAATAGAATAACCAGATA } \\
\text { TGCGGCGCAACGGGTGCTGCG } \\
\text { ACTATCTGGAGATTTAACCATA } \\
\text { TGAATATCCTCCTTAG }\end{array}$ & \\
\hline orf10Mu-F & $\begin{array}{l}\text { GACCTTCCTTACTGATGATGA } \\
\text { AATAAAAATCATACAAAGTI } \\
\text { TTAGAGGATGTTGCCAGTGTAG } \\
\text { GCTGGAGCTGCTTC }\end{array}$ & pKD4 \\
\hline orf10Mu-R & $\begin{array}{l}\text { CATTATCATTIITGCATAAT } \\
\text { GAATGCATTCATTAGAATGCC } \\
\text { TTCCGCCAGGGTTGAAATCATA } \\
\text { TGAATATCCTCCTTAG }\end{array}$ & \\
\hline orf24Mu-F & $\begin{array}{l}\text { TACCCCAGTCAGCCAGCGTTAG } \\
\text { CGCAGTTAAGCCTITAACAGCCAT } \\
\text { TGTCATITCCTCTCGTGTAGG } \\
\text { CTGGAGCTGCTTC }\end{array}$ & pKD4 \\
\hline orf24Mu-R & $\begin{array}{l}\text { AGCGCATTGCCCTGAACAGTATT } \\
\text { TGAAGATGGCCAAAGAGGCC } \\
\text { AGTGAACAGGAGTGATCCATA } \\
\text { TGAATATCCTCCTTAG }\end{array}$ & \\
\hline orf4OMu-F & $\begin{array}{l}\text { AACCTGACATGCGAGAAAGGG } \\
\text { CTTCTCCGTCACGGTIITGTATCG } \\
\text { CGGTAAAAACGTTGTGTGTAGGC } \\
\text { TGGAGCTGCTTC }\end{array}$ & pKD4 \\
\hline orf4OMu-R & $\begin{array}{l}\text { ATTAAGCAGATCATGATTAATTC } \\
\text { CTCCCCACAACATTCAAGCTGATAG } \\
\text { CGGAGATTAATCCATATGAA } \\
\text { TATCCTCCTTAG }\end{array}$ & \\
\hline orf41Mu-F & $\begin{array}{l}\text { ACCTGGATCTGTCTCCCAGGC } \\
\text { CCAATCTGTTGCATGTCTGCTC } \\
\text { ATGATTAATCTCCGCTAGT } \\
\text { GTAGGCTGGAGCTGCTTC }\end{array}$ & pKD4 \\
\hline orf41Mu-R & $\begin{array}{l}\text { GATCTIITITGTGTCAGCAC } \\
\text { AAAATAACCGTAATCCCAA } \\
\text { TACTAATAACAGGGCTTACCC } \\
\text { ATATGAATATCCTCCTAAG }\end{array}$ & \\
\hline iutAMu-F & $\begin{array}{l}\text { CTTCTITAACTCGCTACACA } \\
\text { GCATCTITGGGCTGATIIT } \\
\text { TCCGCCCGTATGGAGGAATAG } \\
\text { TGTAGGCTGGAGCTGCTTC }\end{array}$ & pKD4 \\
\hline iutAMu-R & $\begin{array}{l}\text { CAACTTGGCTGTCAGCGGC } \\
\text { CATGTTGATATCAGCGTACCTाT } \\
\text { GTTGTAAAGGAATACCGGCATAT } \\
\text { GAATATCCTCCTTAG }\end{array}$ & \\
\hline $\mathrm{RT}-d n a E-\mathrm{F}$ & ATGTCGGAGGCGTAAGGCT & dnaE \\
\hline RT-dnaE-R & TCCAGGGCGTCAGTAAACAA & \\
\hline RT-ompA-F & GGTTAGGTCGTATGCCATA & ompA \\
\hline
\end{tabular}

(Continued)
TABLE 2 | Continued

\begin{tabular}{|c|c|c|}
\hline Primer & Sequence $\left(5^{\prime}\right.$ to $\left.3^{\prime}\right)$ & Target gene \\
\hline RT-ompA-R & GTCCAGATCGTCAGTGAT & \\
\hline RT-bor-F & TATTACAGGATGTGCTCAAC & bor \\
\hline RT-bor-R & CCCGAAACGAAGAAATGAT & \\
\hline $\mathrm{RT}-k p s F-\mathrm{F}$ & GGAATGGTGATGGTAGAAGA & kpsF \\
\hline $\mathrm{RT}-k p s F-\mathrm{R}$ & CGTAGCGAATGTCAGAGA & \\
\hline $\mathrm{RT}-k p s E-\mathrm{F}$ & TCTTATCAGGACAACAACAAC & kpsE \\
\hline $\mathrm{RT}-k p s E-\mathrm{R}$ & CCATCAGCGTATTCACTAAC & \\
\hline $\mathrm{RT}-k p s C-\mathrm{F}$ & CGTTATGGCGAATGGAAG & kpsC \\
\hline $\mathrm{RT}-k p s C-\mathrm{R}$ & CGTGGCGTCATAGTAGAT & \\
\hline $\mathrm{RT}-k p s M-\mathrm{F}$ & TAGCAGTATCAGCAATCGT & kpsM \\
\hline RT-kpsM-R & AATCAGTGTCTCAAGCAATG & \\
\hline RT-fur-F & ACGTCAGTGCGGAAGATTTAT & fur \\
\hline RT-fur-R & GATACCAGCGTCGTCAAACT & \\
\hline $\mathrm{RT}$-entA-F & GAGCGAAAGTTACAGGTT & entA \\
\hline $\mathrm{RT}$-entA-R & GGCAACATCCATCACTTC & \\
\hline $\mathrm{RT}-$ fepA-F & GTTGAAGGTCTGGAAGGA & fepA \\
\hline $\mathrm{RT}-f e p A-\mathrm{R}$ & AGCATATAAGTGATGTTATTGGT & \\
\hline RT-iroA-F & TTACGGCTGAAGCGGATTAC & iroA \\
\hline RT-iroA-R & CCTGGCAGTCACGGTAAATAA & \\
\hline RT-iroN-F & GATATTCAGGTCAACGAT & iroN \\
\hline RT-iroN-R & CCAGTTATCTAGCACATAT & \\
\hline RT-iucD-F & TTCTATCGCTTCCTTACA & $i u c D$ \\
\hline $\mathrm{RT}$-iucD-R & ATACAGGTTATTCATATCTTCA & \\
\hline RT-iutA-F & AGCGTGGTGGCGAATAAA & iutA \\
\hline RT-iutA-R & TCCGGTACTCCAGTCAGTATC & \\
\hline RT-irp1-F & GGCGAACCCTGCTATGTATT & irp1 \\
\hline RT-irp1-R & GTCCATGCAGTACCAGCTAAA & \\
\hline RT-irp2-F & ATGGATGCCTCCAGCTTTAC & irp2 \\
\hline RT-irp2-R & AAATCATAGCGGGTGTCGATAG & \\
\hline$R T-f y u A-F$ & ATGCCTATGTGGGATGGAATG & fyuA \\
\hline RT-fyuA-R & CCAGTCATCGGTGGTGTATTT & \\
\hline
\end{tabular}

\section{Sample Preparation and RNA Extraction}

The APEC stain DE142 was grown to an $\mathrm{OD}_{600}$ of 0.6 in LB broth with or without $100 \mu \mathrm{M} 2,2$-dipyridyl (DPD) at $37^{\circ} \mathrm{C}$. Then, the total RNA of DE142 was extracted from $3 \mathrm{ml}$ of bacterial culture using the TRIzol reagent (Invitrogen) isolation protocol, and the obtained RNA was purified using an RNeasy Mini Kit (Qiagen). The RNA concentration was determined using a NanoDrop2000 (Thermo Scientific, USA).

\section{Quantitative Real-Time PCR Assay}

The cDNA was amplified by HiScript II Q RT SuperMix for $\mathrm{qPCR}+\mathrm{gDNA}$ wiper (Vazyme Biotech). According to the instructions of the One Step qRT-PCR SYBR Green Kit (Vazyme Biotech), the mRNA transcription levels were measured. The relative expression levels of the genes were calculated using the $2^{-\Delta \Delta \mathrm{Ct}}$ method. Assays were performed three times. The endogenous reference gene DnaE was used to analyse the bacterial genes quantitatively. 


\section{Construction of the Knockout Mutant and Complement Strains}

The lambda red recombinase system was used to construct the knockout mutants (30) with some modifications. Briefly, the homologous recombination constructs were generated with PCR-purified products with the pKD4 kanamycin resistance gene and 60-nucleotide (nt) homology extensions. The plasmid pCP20 was transferred into the mutant to remove the kanamycin resistance gene. Finally, pCP20 was eliminated by serial subculture in $\mathrm{LB}$ at $42^{\circ} \mathrm{C}$. The mutants were verified by PCR and sequencing. To construct the complementation strains, the coding sequences of genes and their putative promoter regions were amplified using the DE142 strain as a template, followed by independent cloning into pSTV28-MCS.

\section{Growth of Strains in Serum, LB, Iron-Deficient, and Iron-Amended Media}

Chicken serum was obtained from specific-pathogen-free (SPF) chickens. To determine the growth rate in serum, single colonies of the WT and mutant strains were selected and cultured overnight at $37^{\circ} \mathrm{C}$. The bacteria culture were centrifuged and washed twice with PBS, so as to remove the LB media. And then, the $\mathrm{OD}_{600}$ values were adjusted to 1.0 with chicken serum; the chicken serum was subcultured $(1: 20)$ and incubated at $37^{\circ} \mathrm{C}$ with shaking at $180 \mathrm{rpm}$. Assays were performed three times. The optical density at $600 \mathrm{~nm}$ of the bacterial culture was measured every $1 \mathrm{~h}$ over a period of $16 \mathrm{~h}$.

For growth in LB and iron-deficient media, each bacterial suspension was subcultured in LB with or without $100 \mu \mathrm{M} 2,2$ dipyridyl (DPD) according to the proportion of 1:100. Then, the cultures were shaken and incubated at $37^{\circ} \mathrm{C}$. Bacterial growth was determined by measuring the $\mathrm{OD}_{600}$ values every $1 \mathrm{~h}$ over a period of $8 \mathrm{~h}$. Assays were performed three times.

For growth in iron-amended media, $100 \mu \mathrm{M}$ 2,2-dipyridyl (DPD) was added into LB media and mixed well to make it fully bonded to metal ions. Then $70 \mu \mathrm{M} \mathrm{FeCl}_{3}$ was supplemented to this iron-deficient media. The growth curve was measured by the same method.

\section{Competition Assays in LB and Iron-Deficient Media}

The competition experiments were carried out as previously described (31) with some modifications. For competition assays in LB, E. coli strains were grown to log phase, collected and suspended in LB broth. For competition assays in irondeficient medium, the bacteria were suspended with LB liquid medium supplemented with $100 \mu \mathrm{M}$ 2,2-dipyridyl. Then, the wild-type strain DE142A was mixed with its derivative strains DE142 $\operatorname{orf6C}$ or DE142 $\operatorname{orf6} 6 / \operatorname{orf}^{*}\left(1 \times 10^{8} \mathrm{CFU}\right.$ for each strain). The mixture was incubated at $37^{\circ} \mathrm{C}$ for $3 \mathrm{~h}$, and the number of each strain was estimated by counting on LB plates with ampicillin (WT) or chloramphenicol (derivative strains). And plating efficiencies of strains on plates with different antibiotic was measure. The results are shown as the competition index (CI). As the strains were mixed at a 1:1 ratio, the CI represented the value of the number of mutant strains divided by WT. The assay was performed in triplicate with three independent experiments.

\section{Survival in Chicken Serum}

The serum bactericidal assay was performed in a 96-well plate as described previously (32). Briefly, SPF chicken serum was diluted to $50 \%$ with PBS. E. coli strains grown to log phase were collected and washed twice with ice-cold PBS. A dose of $10 \mu \mathrm{L}$ each culture suspension $\left(\mathrm{OD}_{600}=1.0\right)$ was inoculated into a 96-well plate containing $190 \mu \mathrm{L}$ of 50 and $100 \%$ serum. After incubation for $0.5 \mathrm{~h}$ at $37^{\circ} \mathrm{C}$, bacterial numbers were calculated using LB plates. The assay was performed in triplicate with three independent experiments.

\section{Experimental Infection of Chickens via the Air Sacs}

The wild-type strain DE142, mutant strains, and complementation strains were cultured in $\mathrm{LB}$ until the $\mathrm{OD}_{600}$ reached 0.6. After pelleting and washing twice with ice-cold PBS, the bacteria were harvested and resuspended in an appropriate volume of PBS to make the concentration of resuspension reach $2 \times 10^{8} \mathrm{CFU}$.

Forty chickens (7 days old) were divided into five groups and separately challenged with the strains prepared above $\left(2 \times 10^{7}\right.$ CFU for each strain) through the respiratory tract. At $24 \mathrm{~h}$ postinfection, birds were sacrificed by $\mathrm{CO}_{2}$ asphyxiation. The organs of the birds were harvested and homogenized, and the number of bacteria in the lung, heart and blood was measured by plating dilutions of the bacterial suspensions onto LB plates for counting.

\section{Ethics Statement}

All animal experiments conformed to the guidelines of the Association for Assessment and Accreditation of Laboratory Animal Care International. The animal study protocol was approved by the Ethical Committee for Animal Experiments of Nanjing Agricultural University (SYXK(SU)2011-0036), Nanjing, China.

\section{Statistical Analysis}

All statistical analyses were performed by the GraphPad Prism Software package (GraphPad Software, La Jolla, CA, USA). The Mann-Whitney $U$-test was used to analyse the data from in vivo colonization, and the difference in the qRT-PCR data was determined using two-way ANOVA. The rest of the data were analyzed by Student's $t$-test. Figures show mean values. Differences were considered significant at $P<0.05$ and indicated by an asterisk $\left(^{*}\right)$.

\section{Accession Numbers}

The protein sequences of ORF6 and ORF10 were submitted to the GenBank database, and the accession numbers were QGF19612.1 and QGF19616.1, respectively. 


\section{RESULTS}

\section{Analysis of Gene Expression Profiles of Prophage phiv142-3 in Chicken Serum and LB in vitro}

As prophage phiv142-3 increased bacterial survival ability in serum, we analyzed the transcriptional level of the prophage phiv142-3 region in DE142 when grown in chicken serum and LB medium. The results showed that most prophage genes were upregulated in chicken serum, among which orf6 (putative tail fiber protein), orf10 (conserved hypothetical protein), orf 24 (hypothetical protein), orf40 (putative primosomal protein), and orf41 (putative protein) were the 5 most upregulated genes (Supplementary Table 1).

\section{orf6 and orf10 Increased Cell Growth in Chicken Serum}

To probe the impact of individual genes in phiv142-3 on bacterial growth in serum, the 5 most upregulated genes mentioned above were knocked out, and the growth of the mutant and WT strains was observed. The deletion mutants showed similar growth curves in LB (Figure 1A). The orf6 and orf10 knockout mutants showed a slow growth rate and lower yields on these substrates compared with WT after cultivation in serum for $10 \mathrm{~h}(P<0.05)$, while the growth of DE142 $\Delta$ orf24, DE142 $\Delta$ orf 40, DE142 $\Delta$ orf 41 showed no obvious difference in serum (Figure 1B). Therefore, we surmised that orf6 and orf10 conferred the ability of the bacterial strain to grow more rapidly and to obtain higher yields in serum.

\section{orf6 Affected the Growth of APEC DE142 in Iron-Deficient Conditions}

Next, we tried to investigate the reason for the decreased

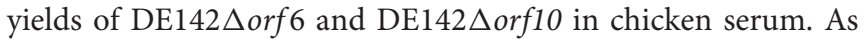
in animal blood, assimilated iron is tightly bound to various proteins, so serum is an iron-deficient condition for bacteria (33). We speculated that orf6 and orf10 might contribute to iron acquisition. To verify our speculation, the growth curves of the mutant and WT strains were analyzed under Fe-deficient conditions. The results showed that the growth rate of the orf6 deletion mutant was slightly decreased compared with that of WT within $6 \mathrm{~h}$. However, after $6 \mathrm{~h}, \mathrm{DE} 142 \Delta$ orf6 showed significantly reduced growth in iron-deficient conditions $(P$ $<0.05)$ compared with WT. And There was no significant difference between the growth rates of WT and DE142 $\Delta$ orf 10 or DE142 $\Delta$ orf6/orf6* in iron-deficient conditions (Figure 2A). Meanwhile, after $\mathrm{FeCl}_{3}$ was added into the iron-deficient media, this difference was disappeared (Figure 2B). Hence, the results indicated that orf6 contributed to the iron acquisition of DE142 in serum.

To further investigate how orf6 influences iron acquisition in chicken serum, the transcription levels of well-known genes related to the acquisition of iron, including enterobactin (entA and its receptor $f e p A$ ), salmochelin (iroB and its receptor iroN), yersiniabactin (irp1, irp2, and its receptor $f y u A$ ) aerobactin ( $i u c D$ and its receptor iutA) and ferric uptake regulation gene fur, were investigated by qRT-PCR. The RNA was isolated from cells grown in iron-deficient medium. Compared with WT, the transcription levels of iutA decreased to $43 \%$ in DE142 $\Delta$ orf6, while the other genes showed no significant differences between

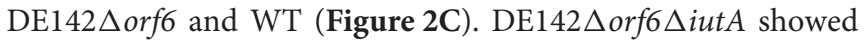
the slowest growth rate (Figure 2A). However, when obtaining plasmid encoded iutA and successful overexpression of iutA (Figure 2D), DE142 $\Delta$ orf6/iutA* grew faster than DE142 $\Delta$ orf6 but still slower than WT in iron-deficient media (Figure 2A). Meanwhile, the transcription level of iutA of DE142 $\Delta$ orf6/orf6* restore to that of WT (Figure 2A). In total, these data indicated that orf6 not only by upregulating the expression of iutA, but also affecting other aspects of the iron-acquisition ability of APEC strain DE142.

\section{orf6 Increased Competitiveness in Iron-Limited Conditions}

The capacity of DE142 $\Delta$ orf6 to compete for growth in LB or iron-deficient media in vitro was compared with that of WT. In bacterial competition assays, we observed that the strains showed similar cell numbers in LB after incubation for $3 \mathrm{~h}$ at $37^{\circ} \mathrm{C}$. However, the number of DE142 $\Delta$ orf6 cells was $\sim 80 \%$ that of WT cells in iron-deficient conditions, with a CI value of 0.78 (Figure 3A, $P<0.05$ ). The competitiveness of complementation strain DE142Aorf6/orf6* was restored to those of the WT (Figure 3B).

\section{The Resistance of the orf10 KO Mutant to Serum Decreased}

Next, we investigated the function of orf10 in serum survival. Assays designed to measure the bactericidal activity of mutant and WT cells in serum were performed. The bactericidal assays showed that the survival of the mutant DE142 $\Delta$ orf 10 was $\sim 60 \%$ $(P<0.01)$ compared with WT in $50 \%$ chicken serum. The results also showed that there were no significant differences in 50 and $100 \%$ chicken serum between WT and DE142 $\Delta$ orf6 (Figure 4A). These results suggested that orf10 enhances the resistance of DE142 to serum.

To further illustrate how orf10 enhances the resistance of DE142 to serum, the transcription levels of several wellknown genes related to serum resistance in E. coli (ompA, bor, and $\mathrm{K} 1$ capsule-related genes $k p s F, k p s E, k p s C$, and $k p s M$ ) (20) were measured by qRT-PCR. RNA was extracted from bacteria growing in LB. The results showed that these genes, except for bor and ompA, were significantly downregulated in DE142 $\operatorname{orf10}(P<0.01)$. The mRNA levels of these genes showed no significant differences between DE142 $\Delta$ orf6 and WT (Figure 4B). This result suggested that the orf10 gene enhanced bacterial resistance to serum by upregulating the expression of $\mathrm{K} 1$ capsule-related genes.

\section{orf6 and orf10 Contributed to Colonization in Chicken Blood and Heart}

To determine whether orf6 and orf10 play a role in serum survival in vivo, animal experiments were carried out to measure the colonization ability of the WT and its derivative strains. 

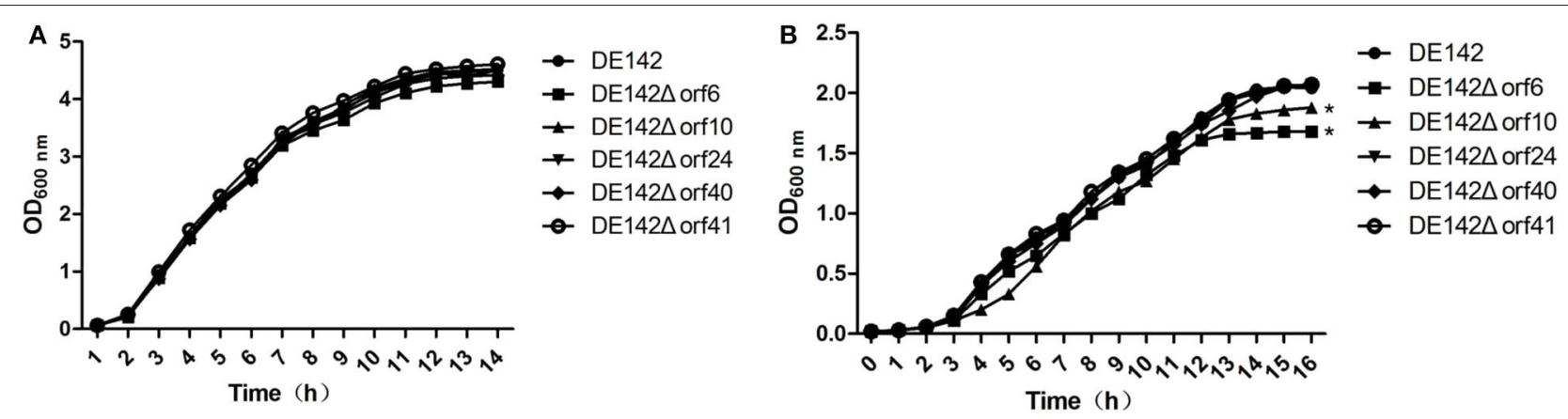

FIGURE 1 | Growth curve of different strains. (A) Growth curves of the wild-type strain DE142 and its derivative mutant strains in LB broth. (B) Growth curves of the wild-type strain DE142 and its derivative mutant strains in SPF chicken serum. Values were the average of three independent experiments $\left({ }^{*} P<0.05\right.$ by unpaired t-test).

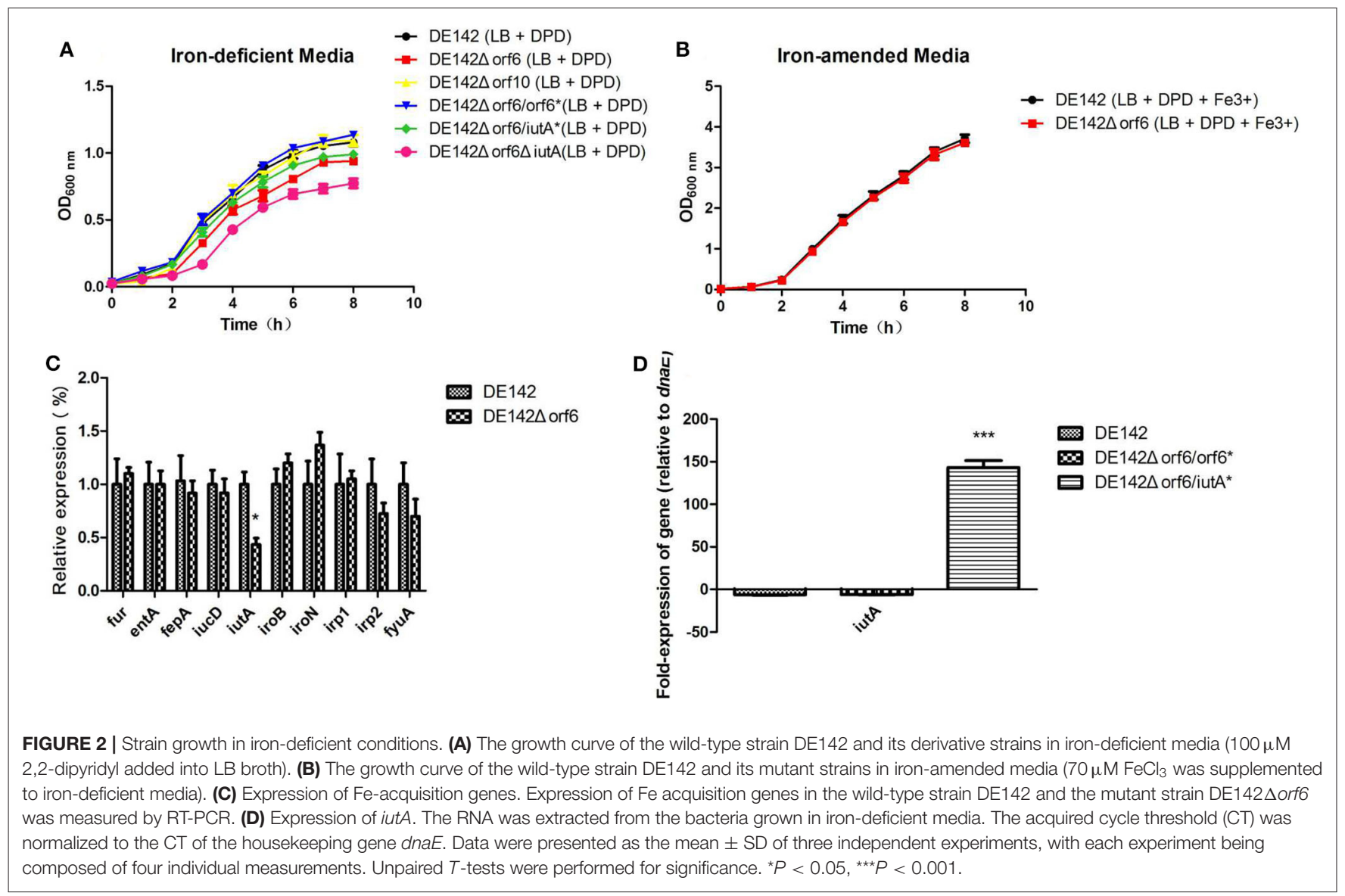

Compared with those challenged with WT, the bacterial loads in blood and heart tissue of chickens challenged with DE142 $\Delta$ orf6 were decreased to 3.9 and $13 \%$, while the bacterial burden in blood and heart from chickens infected with DE142 $\Delta$ orf10 was decreased to 7.2 and $8 \%$, respectively (Figures $5 \mathbf{B}, \mathbf{C}$ ). There were no significant differences in the lung (Figure 5A). The loads of the complementation strain were restored to those of the WT. The results indicated that orf6 and orf10 indeed contributed to the survival of D142 in chicken serum in vivo.

\section{DISCUSSION}

APEC strains cause infection, which leads to huge economic losses in the poultry industry worldwide (34). The ability of APEC strains to resist serum may play an important role in the pathogenesis of avian colibacillosis (21). Thus, improving the understanding of the mechanisms of bacterial resistance to serum would be beneficial to the poultry industry. A previous study in our laboratory revealed that knocking out prophage phiv142-3 

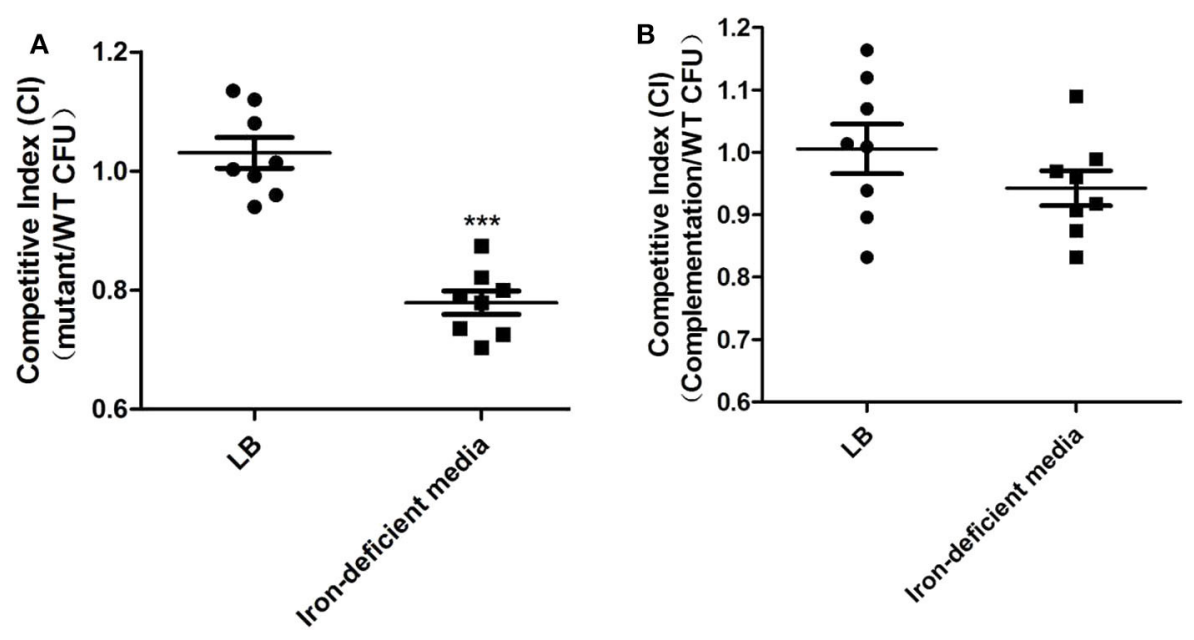

FIGURE 3 | Competition assays. (A) The competition between WT and DE142 $\Delta$ orf6. (B) The competition between WT and DE142 $\Delta$ orf6/orf6 ${ }^{\star}$. Each derivative strain was mixed with the wild-type strain DE142A at a concentration ratio of $1: 1$. After incubation at $37^{\circ} \mathrm{C}$ for $3 \mathrm{~h}$, the number of each strain was estimated by counting on LB plates with ampicillin (WT) or chloramphenicol (derivative strains). The competition index (Cl) represented the final relative numbers of the derivative strain compared to the wild-type strain DE142A. The Mann-Whitney $U$-test was performed for significance, ${ }^{\star * \star} P<0.001$.
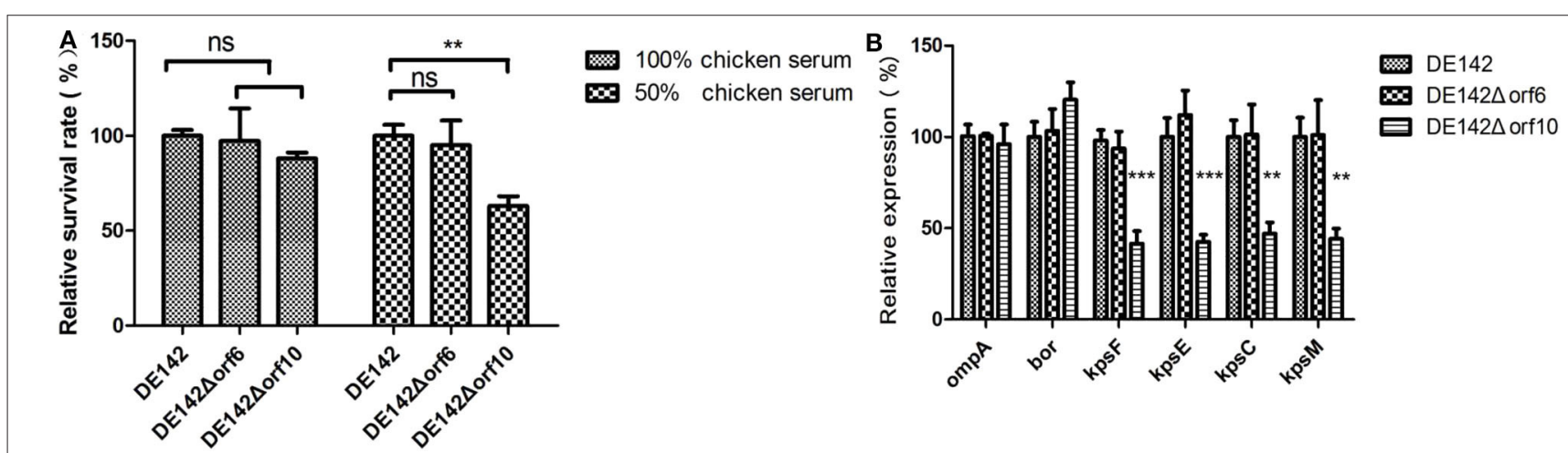

FIGURE 4 | Bacterial resistance to normal chicken serum. (A) The survival rate of the strains in chicken serum. Bacteria were incubated with SPF chicken serum at different dilutions at $37^{\circ} \mathrm{C}$. After $30 \mathrm{~min}$, the remaining number of surviving bacteria was counted on LB plates. In the $100 \%$ chicken serum, the $100 \%$ repesents a $18 \%$ of inoculum of the WT; While in the $50 \%$ chicken serum, the $100 \%$ repesents a $32 \%$ of inoculum of the WT. DE $142 \Delta$ orf 10 showed significantly reduced resistance in $50 \%$ chicken serum. One-way analysis was performed for significance. ${ }^{\star \star} P<0.01$. (B) Quantification of genes related to serum resistance. Expression levels of ompA, bor and $\mathrm{K} 1$ capsule-related genes ( $k p s F, k p s E, k p s C$, and kpsM) in DE142 (WT) and mutant strains were measured by qRT-PCR. The acquired cycle threshold (CT) was normalized to the CT of the housekeeping gene dnaE. Data were presented as the mean \pm SD of three independent experiments, with each experiment being comprised of four individual measurements. Unpaired $T$-tests were performed for significance. ${ }^{\star \star} P<0.01,{ }^{* \star \star} P<0.001$.

from DE142 resulted in a decreased survival rate of the mutant strain in chicken serum (29). In this study, we screened the transcription level of phiv142-3 genes in cells grown in serum and LB by qRT-PCR. Then, several phiv142-3 genes were upregulated when cultured in chicken serum and selected for investigation. Our results indicated that orf6 and orf 10 increased DE142 growth in serum by promoting the acquisition of iron and increasing bacterial resistance to serum, respectively.

In order to identify the genes in phiv142-3 associated with bacterial growth in serum, the transcription level of bacterial genes in LB were compared. Some genes (14/54) in phiv1423 were significantly upregulated when cells were cultured in chicken serum (Supplementary Table 1). The phiv142-3 genes that were significantly upregulated, orf6 ( $2^{2.37}$-fold $)$, orf10 $\left(2^{4.12}\right.$ fold), orf24 ( $2^{1.25}$-fold), orf40 ( $2^{3.68}$-fold), and orf41 ( $2^{4.56}$-fold), were selected, and whether they play roles in bacterial growth in serum was tested. Their different mutants were constructed.

First, we observed that the orf6 and orf10 knockout mutants showed a slower growth rate than the WT in chicken serum, and deletion of orf6 or orf10 didn't influence the transcription of the downstream genes nearby (data not shown). After cultivation in serum for $10 \mathrm{~h}$, the two mutants obtained lower yields $(P$ $<0.01$ ) on these substrates compared with WT (Figure 1B). However, there were no significant differences in growth among the WT and its mutant strains when cultured in LB (Figure 1A). In animal blood, assimilated iron is tightly bound to various 

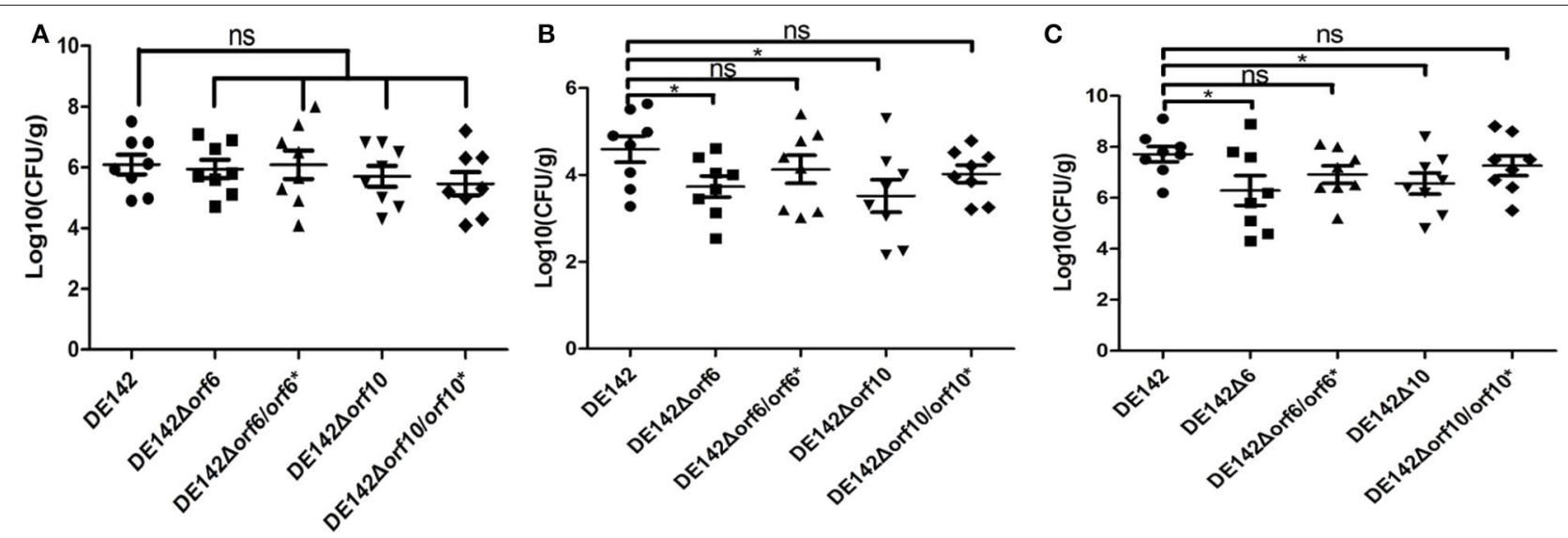

FIGURE 5 | Bacterial colonization during infection in vivo. Infection of chickens via the air sacs with $2 \times 10^{7} \mathrm{CFU}$ of WT DE142, DE142 $\Delta$ orf6, DE142 $\Delta$ orf6/orf6 ${ }^{\star}$, DE142 $\Delta$ orf10, or DE142 $\Delta$ orf10/orf10*. After $24 \mathrm{~h}$ post-infection, bacterial re-isolation from the (A) lung, (B) heart, and (C) blood was quantified by the plate counting method. Each data point represented a sample from an individual chicken. The Mann-Whitney $U$-test was performed for significance, ${ }^{\star} P<0.05$.

proteins, so serum is an iron-deficient condition for APEC (28). Thus, we examined the growth curves of strains in iron-deficient conditions. As shown in Figure 2A, the logarithmic growth phase of the orf6 deletion mutant was significantly decreased compared with that of the WT and the complement strain DE142 $\Delta$ orf6/orf6* was restored to that of WT. Combination with the result in Figure $\mathbf{2 B}$, after $\mathrm{FeCl}_{3}$ was added into the irondeficient media, DE142 $\Delta$ orf6 and WT showed a similar growth rate. It indicated that orf6 affecting the iron-acquisition ability of WT. Next, we determined the transcription level of genes related to the acquisition of iron. The mRNA levels of entA, fepA, iroN, irp1, irp2, fyuA, iucD, and fur in DE142 $\operatorname{orf6}$ showed no significant difference from those in WT. In DE142 $\Delta$ orf6, the transcription levels of iutA decreased to 43\% (Figure 2C, $P<$ 0.05). The iutA protein, which is present on the surface of the cell, is the receptor of the aerobactin siderophore (35). When transfered the plasmid encoding orf6 into DE142 $\Delta$ orf6 resulting in a rise of the transcription level of ituA and growth rate in iron-deficient media (Figures 2A,D). Here, the deletion of orf6 lead to the decreased expression of $i u t A$ and decrease the iron uptake ability of DE142 $\Delta$ orf6. As the overexpresion of $i u t A$ in DE142 $\Delta$ orf6/iutA* still grew slower than WT in iron-deficient media, and We speculated that iutA was an important but not the only reason that orf6 affected the iron-acquisition ability of APEC strain DE142. Furthermore, the interaction between bacteria and the host and the competition for iron are crucially important for the outcome of infection (28). The competition assays also showed that DE142 $\Delta$ orf6 was at a disadvantage when incubated with DE142 in iron-deficient medium (Figure 3). To our knowledge, no study has demonstrated that prophage and iron acquisition are directly connected, but this connection could be mediated by a quorum-sensing system. By sensing available iron in the environment and producing quorum-sensing signals, bacteria can adjust their iron acquisition ability. For instance, after obtaining a plasmid containing a functional copy of LuxS, an $E$. coli strain achieved high cell density under iron-limited conditions (36). Meanwhile, the prophages phiCDHMI and phi3T have been demonstrated to produce quorum sensing precursors $(37,38)$. On the other hand, recent reports have revealed that the tail protein gp138 from bacteriophage $\varphi 92$ contains an iron-binding region (39), and the ORF6 protein in this study has $24 \%$ query coverage with gp138 by blastp on NCBI (Figure 6). The expression of two CJIE1 prophage tail proteins has been reported to be upregulated in sheep blood (40), indicating that prophage tail proteins may play a role in bacterial growth in animal blood. Here, ORF6 is a putative tail fiber protein based on genome annotation. Whether ORF6 acts as a "siderophore" or influences iron acquisition by affecting quorum-sensing signals needs to be further studied.

The bactericidal assays performed with SPF chicken serum revealed that the resistance of DE142 $\Delta$ orf10 to serum was decreased. The $\mathrm{K} 1$ capsule is reported to participate in bacterial resistance to serum (23). Here, K1 capsule-related genes were found to be downregulated nearly 2-fold in DE142 $\Delta$ orf10 (Figure 4B). These results were consistent with the previous observation that DE142 $\Delta$ orf10 showed a slower growth rate when grown in serum at first, and as time passed, the mutant obtained lower yields on these substrates compared with WT after cultivation in serum for $12 \mathrm{~h}$ (Figure 1B, $P<0.01$ ). Paniagua-Contreras, G. L found that the Lambda prophage in $E$. coli could increase host bacteria survival ability in human serum (41). Prophages not only encode proteins directly participating in serum bactericidal effects but also alter the traits of their host bacteria (7). Here, orf10 contributed to serum resistance by influencing the expression of K1 capsulerelated genes.

APEC invades the host through the respiratory tract, followed by bacteraemia; thus, serum resistance appears to be an important factor for APEC infection (21). In vivo studies revealed that the ability of the mutant strains DE142 $\Delta$ orf6 and DE142 $\Delta$ orf10 to colonize chicken heart and blood was significantly decreased compared with that of the WT 


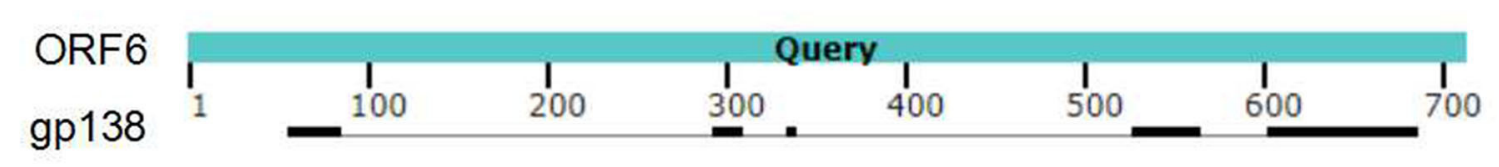

FIGURE 6 | Analyses of the sequences of ORF6 and gp138. Identical regions were highlighted in black.

strain (Figures 5B,C). These results corresponded with the

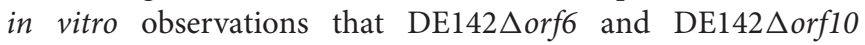
obtained lower yields when cultured in chicken serum (Figure 1B). However, orf6 and orf10 did not affect the bacterial colonization ability in the lung (Figure 5A) or adhesion ability to DF-1 cells (data not shown). The results indicated that orf6 and orf 10 did not affect bacterial adhesion ability but played a role in bacterial resistance to serum in vivo.

In this study, some genes in phiv142-3 were screened and identified to be associated with bacterial growth in serum. Deletion of the prophage genes orf6 and orf10 led to a decrease in the number of bacteria when incubated with serum and decreased the ability of the bacterial strain to colonize chicken blood and heart tissue. Our results suggested that orf6 affects these aspects by improving the uptake ability of iron, while orf10 increased serum resistance by upregulating K1 capsule-related genes.

\section{CONCLUSION}

The present work investigated the roles of genes in prophage phiv142-3 associated with bacterial growth in serum in vitro and in vivo. Through qRT-PCR, orf6, and orf10 in phiv1423 attracted our attention because they were upregulated in chicken serum compared with LB. The orf6 and orf 10 knockout mutants showed a slower growth rate than WT in chicken serum. Meanwhile, compared with WT, the bacterial loads in blood and heart tissue of the chickens challenged with DE142 $\Delta$ orf6 or DE142 $\Delta$ orf 10 were also significantly decreased in vivo. Furthermore, we explored the deeper reason for these phenomena. On the one hand, the growing ability of DE142 $\Delta$ orf 6 was attenuated in iron-deficient media. Further study found iutA was an important but not the only reason that orf6 affected the iron-acquisition ability of APEC strain DE142. On the other hand, bactericidal assays performed with SPF chicken serum revealed that the resistance of DE142 $\Delta$ orf 10 to serum was decreased. The K1 capsule is reported to participate in bacterial resistance to serum. Here, K1 capsule-related genes were found to be downregulated nearly 2 -fold in DE142 $\Delta$ orf 10 . Thus, we speculate that orf6 affects APEC strain DE142 growth in serum by improving the uptake ability of iron, while orf 10 increases the resistance of DE142 to serum by upregulating K1 capsule-related genes.

\section{DATA AVAILABILITY STATEMENT}

The protein sequences of ORF6 and ORF10 were submitted to the GenBank database, 198 and the accession numbers were QGF19612.1 and QGF19616.1, respectively.

\section{ETHICS STATEMENT}

All animal experiments conformed to the guidelines of the Association for Assessment and Accreditation of Laboratory Animal Care International. The animal study protocol was approved by the Ethical Committee for Animal Experiments of Nanjing Agricultural University (SYXK(SU)2011-0036), Nanjing, China.

\section{AUTHOR CONTRIBUTIONS}

JD concevied and designed the study. DL was responsible for experimental operation and drafted the manuscript. XQ, $\mathrm{XL}$, and YS gave experimental help. JR, FX, and QL gave article modification help. FT provided valuable suggestions of the manuscript. All authors approved the final version of the manuscript.

\section{FUNDING}

This work was funded by the Natural Science Foundation of Jiangsu Province (BK20180075), Youth Foundation of the National Natural Science Foundation of China (Grant 31402213), and Science and Technology innovation Plan of Shanghai (19391902000).

\section{ACKNOWLEDGMENTS}

Authors are grateful to all of the staff at Microbiology laboratory, Veterinary medicine, Nanjing Agricultural University, China. We also gratefully acknowledge the members of School of Medical Instrument and Food Engineering, University of Shanghai for Science and Technology, Shanghai, China.

\section{SUPPLEMENTARY MATERIAL}

The Supplementary Material for this article can be found online at: https://www.frontiersin.org/articles/10.3389/fvets. 2020.588708/full\#supplementary-material 


\section{REFERENCES}

1. Bettarel Y, Sime-Ngando T, Amblard C, Laveran H. A comparison of methods for counting viruses in aquatic systems. Appl Environ Microbiol. (2000) 66:2283-9. doi: 10.1128/AEM.66.6.2283-2289.2000

2. Casjens S. Prophages and bacterial genomics: what have we learned so far? Mol Microbiol. (2003) 49:277-300. doi: 10.1046/j.1365-2958.2003.03580.x

3. Dedrick RM, Jacobs-Sera D, Bustamante CA, Garlena RA, Mavrich TN, Pope $\mathrm{WH}$, et al. Prophage-mediated defence against viral attack and viral counterdefence. Nat Microbiol. (2017) 2:16251. doi: 10.1038/nmicrobiol.2016.251

4. Bondy-Denomy J, Qian J, Westra ER, Buckling A, Guttman DS, Davidson AR, et al. Prophages mediate defense against phage infection through diverse mechanisms. ISME J. (2016) 10:2854-66. doi: 10.1038/ismej.2016.79

5. Fogg PC, Allison HE, Saunders JR, McCarthy AJ. Bacteriophage lambda: a paradigm revisited. J Virol. (2010) 84:6876-9. doi: 10.1128/JVI.02177-09

6. Fu Y, Wu Y, Yuan Y, Gao M. Prevalence and diversity analysis of candidate prophages to provide an understanding on their roles in Bacillus thuringiensis. Viruses. (2019) 11:388. doi: 10.3390/v11040388

7. Taylor VL, Fitzpatrick AD, Islam Z, Maxwell KL. The diverse impacts of phage morons on bacterial fitness and virulence. Adv Virus Res. (2019) 103:1-31. doi: 10.1016/bs.aivir.2018.08.001

8. Tsao YF, Taylor VL, Kala S, Bondy-Denomy J, Khan AN, Bona D, et al. Phage morons play an important role in Pseudomonas aeruginosa phenotypes. $J$ Bacteriol. (2018) 200:e00189-18. doi: 10.1128/JB.00189-18

9. Su LK, Lu CP, Wang Y, Cao DM, Sun JH, Yan YX. Lysogenic infection of a Shiga toxin 2-converting bacteriophage changes host gene expression, enhances host acid resistance and motility. Mol Biol. (2010) 44:6073. doi: 10.1134/S0026893310010085

10. Cao D, Ji W, Fu Q, Lu C, Wang H, Sun J, et al. Escherichia coli nfuA is essential for maintenance of Shiga toxin phage Min27 lysogeny under iron-depleted condition. FEMS Microbiol Lett. (2015) 362:fnv149. doi: 10.1093/femsle/fnv149

11. Tang F, Zhang W, Lu C. Lysogenic Streptococcus suis isolate SS2-4 containing prophage SMP showed increased mortality in zebra fish compared to the wildtype isolate. PLoS ONE. (2013) 8:e54227. doi: 10.1371/journal.pone.0054227

12. Wang X, Kim Y, Ma Q, Hong SH, Pokusaeva K, Sturino JM, et al. Cryptic prophages help bacteria cope with adverse environments. Nat Commun. (2010) 1:147. doi: 10.1038/ncomms1146

13. Barondess JJ, Beckwith J. bor gene of phage lambda, involved in serum resistance, encodes a widely conserved outer membrane lipoprotein. J Bacteriol. (1995) 177:1247-53. doi: 10.1128/JB.177.5.1247-1253.1995

14. Lynne AM, Skyberg JA, Logue CM, Nolan LK. Detection of Iss and Bor on the surface of Escherichia coli. J Appl Microbiol. (2007) 102:6606. doi: 10.1111/j.1365-2672.2006.03133.x

15. Yu ZC, Chen XL, Shen QT, Zhao DL, Tang BL, Su HN, et al. Filamentous phages prevalent in Pseudoalteromonas spp. confer properties advantageous to host survival in Arctic sea ice. ISME J. (2015) 9:87181. doi: 10.1038/ismej.2014.185

16. Rabinovich L, Sigal N, Borovok I, Nir-Paz R, Herskovits AA. Prophage excision activates Listeria competence genes that promote phagosomal escape and virulence. Cell. (2012) 150:792-802. doi: 10.1016/j.cell.2012. 06.036

17. Davies EV, James CE, Kukavica-Ibrulj I, Levesque RC, Brockhurst MA, Winstanley C. Temperate phages enhance pathogen fitness in chronic lung infection. ISME J. (2016) 10:2553-5. doi: 10.1038/ismej.2016.51

18. Li XY, Lachnit T, Fraune S, Bosch TCG, Traulsen A, Sieber M. Temperate phages as self-replicating weapons in bacterial competition. J R Soc Interface. (2017) 14:20170563. doi: 10.1098/rsif.2017.0563

19. Dho-Moulin M, Fairbrother JM. Avian pathogenic Escherichia coli (APEC). Vet Res. (1999) 30:299-316.

20. Mellata M, Dho-Moulin M, Dozois CM, Curtiss R, 3rd, Lehoux B, Fairbrother JM. Role of avian pathogenic Escherichia coli virulence factors in bacterial interaction with chicken heterophils and macrophages. Infect Immun. (2003) 71:494-503. doi: 10.1128/IAI.71.1.494-503.2003

21. Li G, Tivendale KA, Liu P, Feng Y, Wannemuehler Y, Cai W. et al. Transcriptome analysis of avian pathogenic Escherichia coli O1 in chicken serum reveals adaptive responses to systemic infection. Infect Immun. (2011) 79:1951-60. doi: 10.1128/IAI.01230-10
22. Wojnicz D, Cisowska A. Composition of the outer membrane proteins of Escherichia coli strains in relation to serum susceptibility after exposure to subinhibitory concentrations of amikacin and ciprofloxacin. Int J Antimicrob Agents. (2009) 33:579-82. doi: 10.1016/j.ijantimicag.2008. 12.006

23. Yousuf FA, Rafiq S, Siddiqui R, Khan NA. The role of genomic islands in Escherichia coli $\mathrm{K} 1$ interactions with intestinal and kidney epithelial cells. Microb Pathog. (2016) 93:145-51. doi: 10.1016/j.micpath.2016.02.002

24. Arezes J, Jung G, Gabayan V, Valore E, Ruchala P, Gulig PA, et al. Hepcidininduced hypoferremia is a critical host defense mechanism against the siderophilic bacterium Vibrio vulnificus. Cell Host Microbe. (2015) 17:4757. doi: 10.1016/j.chom.2014.12.001

25. Reddy YS, Srivalliputturu SB, Bharatraj DK. The effect of lead $(\mathrm{Pb})$ exposure and iron (Fe) deficiency on intestinal lactobacilli, E. coli and yeast: a study in experimental rats. J Occup Health. (2018) 60:47584. doi: 10.1539/joh.2017-0267-OA

26. Garenaux A, Houle S, Folch B, Dallaire G, Truesdell M, Lepine F, et al. Avian lipocalin expression in chickens following Escherichia coli infection and inhibition of avian pathogenic Escherichia coli growth by Ex-FABP. Vet Immunol Immunopathol. (2013) 152:156-67. doi: 10.1016/j.vetimm.2012. 09.018

27. Heinemann IU, Jahn M, Jahn D. The biochemistry of heme biosynthesis. Arch Biochem Biophys. (2008) 474:238-51. doi: 10.1016/j.abb.2008.02.015

28. Ratledge C, Dover LG. Iron metabolism in pathogenic bacteria. Annu Rev Microbiol. (2000) 54:881-941. doi: 10.1146/annurev.micro.54.1.881

29. Li D, Tang F, Xue F, Ren J, Liu Y, Yang D, et al. Prophage phiv1423 enhances the colonization and resistance to environmental stresses of avian pathogenic Escherichia coli. Vet Microbiol. (2018) 218:707. doi: 10.1016/j.vetmic.2018.03.017

30. Datsenko KA, Wanner BL. One-step inactivation of chromosomal genes in Escherichia coli K-12 using PCR products. Proc Natl Acad Sci USA. (2000) 97:6640-5. doi: 10.1073/pnas.120163297

31. Xiong L, Ling J, Gao Q, Zhou Y, Li T, Gao S, et al. Construction of iucB and iucBiutA mutants of avian pathogenic Escherichia coli and evaluation of their pathogenicity. Vet Microbiol. (2012) 159:420-31. doi: 10.1016/j.vetmic.2012. 04.024

32. Wang S, Dai J, Meng Q, Han X, Han Y, Zhao Y, et al. DotU expression is highly induced during in vivo infection and responsible for virulence and Hcpl secretion in avian pathogenic Escherichia coli. Front Microbiol. (2014) 5:588. doi: 10.3389/fmicb.2014.00588

33. Miethke M, Marahiel MA. Siderophore-based iron acquisition and pathogen control. Microbiol Mol Biol Rev. (2007) 71:41351. doi: 10.1128/MMBR.00012-07

34. Vounba P, Kane Y, Ndiaye C, Arsenault J, Fairbrother JM, Alambedji RB. Molecular characterization of Escherichia coli isolated from chickens with colibacillosis in Senegal. Foodborne Pathog Dis. (2018) 15:51725. doi: 10.1089/fpd.2017.2394

35. Murakami K, Fuse H, Takimura O, Inoue H, Yamaoka Y. Cloning and characterization of the iutA gene which encodes ferric aerobactin receptor from marine Vibrio species. Microbios. (2000) 101:137-46.

36. Fong KP, Gao L, Demuth DR. luxS and $\operatorname{arcB}$ control aerobic growth of Actinobacillus actinomycetemcomitans under iron limitation. Infect Immun. (2003) 71:298-308. doi: 10.1128/IAI.71.1.298-308. 2003

37. Hargreaves KR, Kropinski AM, Clokie MR. What does the talking?: quorum sensing signalling genes discovered in a bacteriophage genome. PLOS ONE. (2014) 9:e85131. doi: 10.1371/journal.pone.0085131

38. Erez Z, Steinberger-Levy I, Shamir M, Doron S, Stokar-Avihail A, Peleg Y, et al. Communication between viruses guides lysis-lysogeny decisions. Nature. (2017) 541:488-93. doi: 10.1038/nature21049

39. Browning C, Shneider MM, Bowman VD, Schwarzer D, Leiman PG. Phage pierces the host cell membrane with the ironloaded spike. Structure. (2012) 20:326-39. doi: 10.1016/j.str.2011. 12.009

40. Clark CG, Chong PM, McCorrister SJ, Simon P, Walker M, Lee DM, et al. The CJIE1 prophage of Campylobacter jejuni affects protein expression in growth media with and without bile salts. BMC Microbiol. (2014) 14:70. doi: 10.1186/1471-2180-14-70 
41. Vaca-Pacheco S, Piedra-Ibarra E, Arenas-Aranda D, Paniagua-Contreras GL. Lambda prophage decreases Escherichia coli sensitivity to human serum bactericidal effect. Rev Latinoam Microbiol. (1993) 35:71-5.

Conflict of Interest: The authors declare that the research was conducted in the absence of any commercial or financial relationships that could be construed as a potential conflict of interest.
Copyright (C) 2020 Li, Qian, Liu, Sun, Ren, Xue, Liu, Tang and Dai. This is an open-access article distributed under the terms of the Creative Commons Attribution License (CC BY). The use, distribution or reproduction in other forums is permitted, provided the original author(s) and the copyright owner(s) are credited and that the original publication in this journal is cited, in accordance with accepted academic practice. No use, distribution or reproduction is permitted which does not comply with these terms. 